\title{
Prospects brighten around the red planet
}

Tony Reichhardt, Washington.

Planetary scientists are revelling in a flood of fresh data from Mars. A second NASA rover landed on the planet's surface on 24 January, the first seems to be on the mend after a computer glitch, and the European Space Agency's (ESA's) Mars Express has begun transmitting spectacular photographs and spectral data from martian orbit.

NASA's Opportunity spacecraft, which came down in a rocky plain known as Meridiani Planum, rolled to a stop inside a shallow, 20-metre-wide crater that partially blocked its view of the horizon. But once the rover's systems are checked out, project engineers predict that it will easily get out of the crater. The terrain at Meridiani is different from any previous landing site on Mars, with darker soil and at least one exposed outcrop that may offer a rare glimpse of martian bedrock.

The two rovers are searching for geological evidence of water, past or present, on Mars. The first spacecraft, Spirit, set down in what scientists believe is a dried-up lake bed, whereas the Meridiani site contains an iron mineral - haematite - that forms in the presence of water on Earth.

Spirit went silent on 20 January just as it was about to begin its first close-up studies of a rock. After a nervous 24 hours without radio contact, controllers at NASA's Jet

\section{Photo express}

Quirin Schiermeier, Munich

Spectacular pictures from the European Space Agency's Mars Express mission are being beamed back to Earth each day now. Their beauty and clarity are thrilling geologists - and laying the foundations for the richest database ever gathered of any planet, even our own.

The pictures are the product of the High Resolution Stereo Camera (HRSC). The $€ 70$ million (US\$87-million) instrument was originally developed by scientists at the German Space Agency for Russia's failed Mars '96 mission and has been updated for Mars Express.

Digital processing helps to create the stunning three-dimensional views, but instrument builders say that the pictures are 'real'. Like a normal camera, the HRSC takes photographs through a single lens, which is pointed at the planet's surface each time Mars Express's elliptical orbit reaches its lowest altitude, about 250 kilometres.

Behind the lens, however, is a complex system of parallel sensors, which are sensitive to red, green, blue and near-infrared light. By shooting points on the planet's surface from three different perspectives - forwards, downwards and backwards - the camera collects information that is then processed back on Earth to create a digital model of the terrain with a spatial resolution of 10 metres per pixel or less.

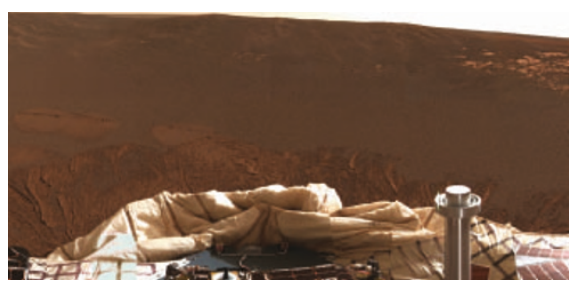

The Mars probe Opportunity prepares to climb the crater wall near its landing spot.

Propulsion Laboratory in Pasadena, California, regained command of the spacecraft. They determined the probable cause of the blackout - a problem with onboard computer memory caused by handling too many data files. Although it may take a couple of weeks to debug the software and check out onboard systems, project managers are optimistic that Spirit will be able to resume exploration. Project scientist Steven Squyres of Cornell University, Ithaca, New York, said the team had anticipated before the mission that such glitches could interrupt science operations as often as one day out of three.

ESA's Mars Express, meanwhile, is nearing the end of its checkout phase in orbit, with all instruments working perfectly, according to project scientist Agustin Chicarro. ESA researchers last week unveiled the first pictures from the orbiter's main camera (see below), as well as data from other instruments. A combined camera and infrared spectrometer called OMEGA has produced the first significant scientific results from the mission: direct detection of water ice and carbon dioxide ice in the planet's south polar cap.

Although some scientists have downplayed the significance of this result, past telescopes and satellites saw only atmospheric water vapour or hydrogen on the martian surface. The direct detection of water molecules in the polar caps is "obviously not a surprise - it's what everybody expected", says Chicarro. But confirming it is a significant step, he says.

Unlike the pictures from the twin NASA rovers, data and images from Mars Express will at first belong to the ESA scientists who built the instruments. After six months, they will be placed in a public archive - an arrangement similar to that for the Mars Observer Camera now orbiting the planet on NASA’s Mars Global Surveyor.

Malin Space Science Systems of San Diego, California, which built that camera, last week released a picture showing the Spirit rover, its parachute and other cast-off hardware as bright dots on the martian surface - the clearest image ever taken of a planetary lander from orbit.

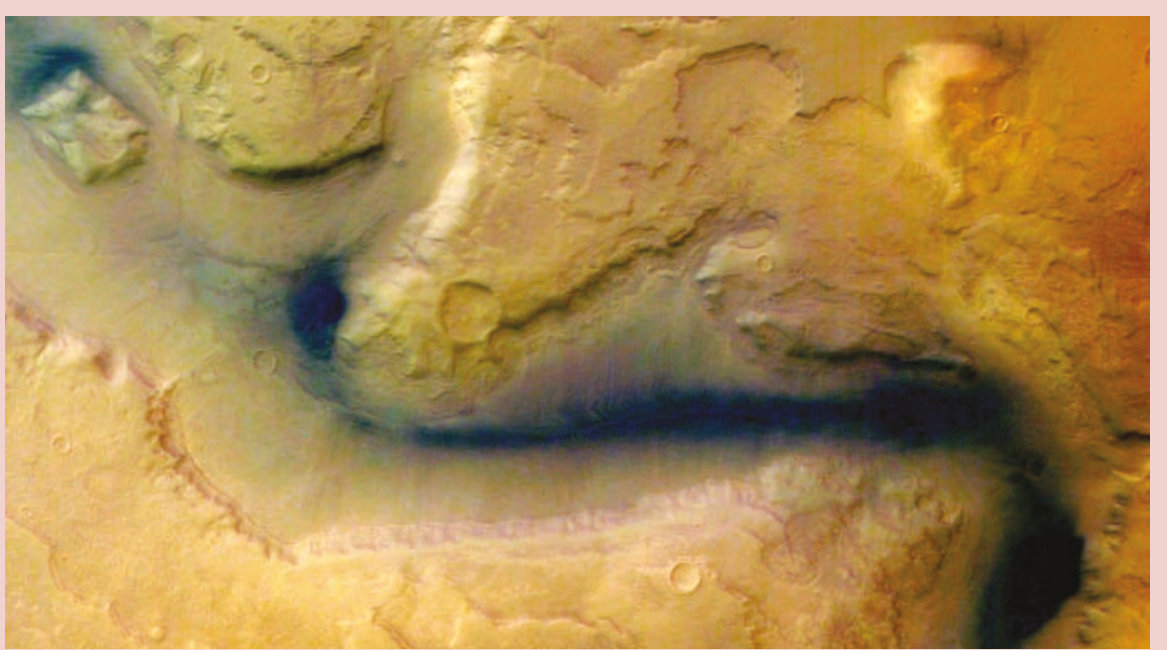

Past evidence: Reull Vallis, a channel once formed by flowing water, as seen from Mars Express.

As of 23 January, after 10 days of observation, 2 million square kilometres - or about $2 \%$ of the Mars surface - had been surveyed. After some 5,000 flyovers, which would take a maximum of four Earth years to complete, the topography of Mars would have been surveyed more comprehensively than that of Earth, where military considerations prevent complete topographical surveys from reaching public databases.

"We can clearly make out slopes and valleys that were almost certainly eroded by running water," says Gerhard Neukum, a planetary scientist at the Free University in Berlin, and principal investigator on the HRSC project. "Moreover, we have found moraines, which proves there must have been glaciers on Mars."

The camera might also be able to spot the large white parachutes of the Beagle 2 lander, which went missing on 25 December last year. But so far the search has been in vain. "There is dust and lack of contrast where we assume it must be," says Neukum. 